\title{
The Design of Surface Acoustic Wave Receiving Circuit Based on Piezoelectric Transducer
}

\author{
Hao Jiang, Fuqiang Zhang, Zhao Guan \\ Shanghai University of Engineering Science, Shanghai, China \\ Email: mmyjh_123@163.com
}

How to cite this paper: Jiang, H., Zhang, F.Q. and Guan, Z. (2018) The Design of Surface Acoustic Wave Receiving Circuit Based on Piezoelectric Transducer. Open Access Library Journal, 5: e4829. https://doi.org/10.4236/oalib.1104829

Received: August 8, 2018

Accepted: September 8, 2018

Published: September 11, 2018

Copyright $\odot 2018$ by authors and Open Access Library Inc.

This work is licensed under the Creative Commons Attribution International License (CC BY 4.0).

http://creativecommons.org/licenses/by/4.0/

\section{cc) (7) Open Access}

\begin{abstract}
A type of Surface Acoustic Wave (SAW) receiving circuit with high stability is introduced in this paper on the basis of analyzing common surface acoustic wave receiving circuits. The circuit design is based on receiving transducer and the center frequency is $1 \mathrm{MHz}$. This circuit includes pre-amplification electric circuit with high input impedance, automatic gain amplifying circuit based on VCA810, tertiary amplifying circuit, filter circuit and automatic voltage comparison circuit. Receiving gate is used to preventing transmission pulse interferes the receipt signal. For better detecting the arrival time of receipt signal, voltage comparing circuit is served as examining the receipt signal, and measurement accuracy of acoustic transit time is improved substantially. By contrast, amplification in three levels improves the circuit's working stability. In addition, the circuit has the advantage of simple structure, signal stability and low manufacturing cost.
\end{abstract}

\section{Subject Areas}

Electric Engineering

\section{Keywords}

SAW, Transducer, Receiving Circuit, Comparing Circuit, VCA810

\section{Introduction}

Surface Acoustic Wave (SAW) is a kind of elastic wave which propagating along the elastic material surface and the propagated energy is concentrated near the surface. SAW is widely used in fields of automation, biomedicine, chemical industry, environment monitor and reconnaissance and so on [1]. And the frequency range of SAW filter is ranging from $10 \mathrm{MHz}$ to $3 \mathrm{GHz}$, and it possesses the characteristic of low output impedance, high dependability, and small in size; 
and it can achieve a variety of complex functions [2] [3] [4].

Many researchers studied the structures and functions of SAW receiving circuits. Gao et al. [5] designed a receiving circuit with IQ demodulator chip, low-noise baseband amplifier, ADC driver, high-speed ADC, FPGA and SRAM, combined with transmitting circuit to constitute a SAW RFID system. Zhou et al. [6] make use of the method of lock-in amplifying to pick up the weak signal from noise, they solve the problem on amplifying the output signal of SAW gyroscope. Kim et al. [7] introduces the transmission or out of band blocker signal canceller architectures in receiver systems. Owaki [8] designed a type of device includes a piezoelectric substrate and an IDT electrode provided on the piezoelectric substrate. Funami et al. [9] made a communication equipment includes SAW resonator, filter and duplexer. Lei et al. [10] fabricated a device with $\mathrm{U}$ wave band $(860 \mathrm{MHz})$ frequency modulation wireless microphone transmitter and receiver and it can achieve in the wide use of wireless amplifier system in multimedia classrooms.

When acoustic emission transducer sends a sound wave signal, the signal propagates through media that received by receiving transducer. Whereas the cause of sound intensity diminishing constantly depends on impurities and energy consumption within media. And the signal must be stable and reliable before it reaches to detection circuit so as to achieve the accurate measurement. In this paper, this design mainly includes pre-amplification electric circuit, automatic gain amplifying circuit, tertiary amplifying circuit, filter circuit and automatic voltage comparison circuit. In comparison with SAW receiving circuits designed by previous scholars, the amplifier circuit is divided into three parts and the first and the third section is fixed-proportion amplified, and intermediate section is auto-gain control amplifier used to improve measurement accuracy. In addition, automatic voltage comparison circuit can examine the receipt signal with effect.

\section{Amplifying Circuit}

In general, the acoustic wave signal received by transducer is weak and even only up to a few millivolts. However, the amplitude of sampled ADC signal is $5 \mathrm{~V}$, thus it is necessary to amplify sampled signal. The circuit is designed to three-stage amplifier circuit, the first-stage and third-stage is fixed gain amplifier, and the second-stage is automatic gain amplifying circuit, and the gain of whole amplifying circuits could be adjusted by changing the chip VCA810's terminal voltage, thereby bringing output signal to specified amplitude.

\section{1) Pre-amplification electric circuit with high input impedance}

The main function of this circuit is impedance matching and amplifying the receiving signals. The impedance of acoustic wave transducer is larger than $10^{6} \Omega$, so the plain amplifier is hard to match with it unless using amplifier with MOS structure in order to improve input impedance. TL031 is specified with a minimum and a maximum input voltage that, if exceed an either input, could 
cause the device to malfunction. Because of the extremely high input impedance and resulting low bias current requirements, and TL031 is well suited for low-level signal processing. Therefore, TL031 is selected as high input impedance operational amplifier and differential input stage is composed of JFET, the input impedance can reach up to $10^{12} \Omega$. The chip TL031 adopts same phase amplify and the stage magnification times is $A=1+R_{\mathrm{f}} / R_{1}=11$, the circuit of high input impedance as shown in Figure 1.

\section{2) Automatic gain amplifying circuit}

Since sonic sensor's exciting energy change with energy consumption and the received amplitude could also make a difference. And if ordinary integrated operational amplifier is instead, amplitude identification method used in acoustic receiving may appear misconception phenomenon. Thus in order to create a similar amount of magnified pulse signal's amplitude, automatic gain amplifying circuit should be eligible to amplifying signal and this type of functionality is usually realized by gain-programmed amplifier. Its working principle is sustain the magnified receipt signal's peak sampling for long and the signal is used to control the gain-programmed amplifier's amplification after A/D conversion, the output power can be kept stable finally. The deficiency of gain-programmed amplifier comprising so many switches and the circuit design is complex, worse still, the ever-changing magnification may contribute to circuit working instability.

The chip VCA810 produced by the United States is used as Auto Gain Control. It is a DC-coupled, continuously variable and voltage-controlled gain integrated amplifier with low-noise gain adjustable bandwidth and the frequency of bandwidth is $25 \mathrm{MHz}$. A linear correlation is found between the gain and control voltage, the slew rate is $300 \mathrm{~V} / \mu \mathrm{s}$. The device provides a differential input to single output conversion with a high-impedance gain over $-40 \mathrm{~dB}$ to $40 \mathrm{~dB}$ range linear in $\mathrm{dB} / \mathrm{V}$. In comparison with $\mathrm{AD} 603$, the noise control is superior to AD603 although frequency of bandwidth is lower than it. The external structure of VCA810 is shown in Figure 2.

Pin1: Non-inverting input

Pin2: Ground

Pin3: Gain control

Pin4: No connect

Pin5: Output

Pin6: Positive supply

Pin7: Negative supply

Pin8: Inverting input

As shown in Figure 3, the VCA810 is a high gain adjustment range, wideband, voltage amplifier with a voltage-controlled gain. The basic voltage amplifier responds to the control of an internal gain-control amplifier. At the input, the circuit presents a high impedance of a differential stage, permitting flexible input impedance machining. The gain voltage $V_{C}$ controls the amplifier gain magnitude through a high-speed control circuit. The gain polarity can be either 


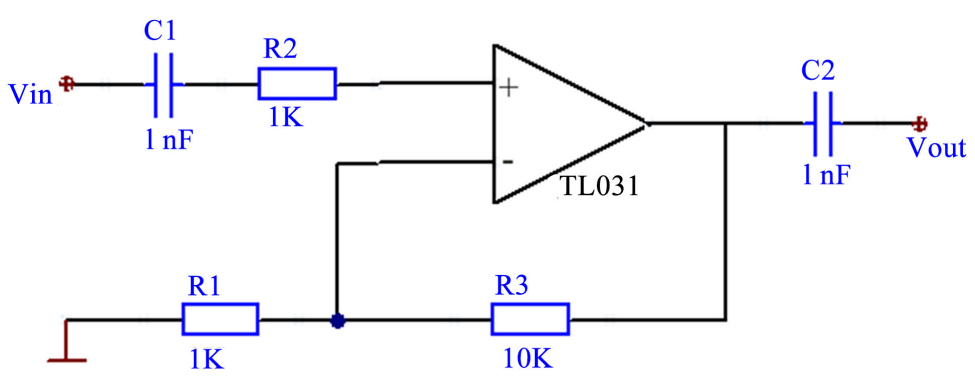

Figure 1. Pre-amplification electric circuit.

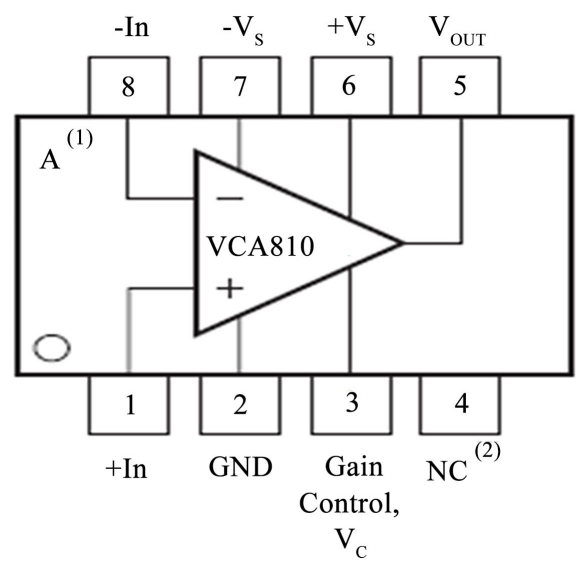

Figure 2. external structure of VCA810.

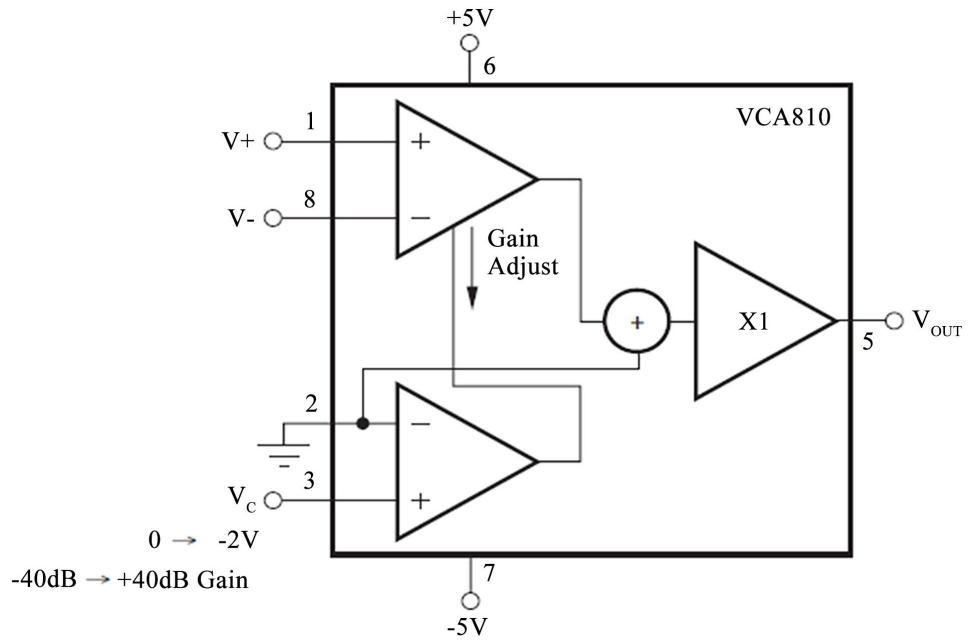

Figure 3. Functional block diagram.

inverting or non-inverting, depends on amplifier input driven by the input signal and the control voltage $V_{C}$ varies the amplifier gain according to the exponential relationship:

$$
G_{(V / V)}=10^{-2\left(V_{c}+1\right)}
$$

Figure 4 shows the configuration on the basis of the circuit's electrical characteristics and typical characteristics. In this circuit, the input impedance is set 
to $50 \Omega$ with a resistance to ground. To eliminating bias current, a $25 \Omega$ resistance is used on the $\mathrm{V}$-input. And the power bypassing is composed of two capacitors on each supply pin, the one small ceramic capacitor is used for high-frequency decoupling, the one large electronic capacitor is used in low frequencies. Notably, the resistances $R_{\mathrm{s}}$ and $R_{\mathrm{t}}$ are connected to ground both on inverting and non-inverting input, and the matching DC source impedance will reduce the error of input offset voltage for furthest.

\section{3) Tertiary amplifying circuit}

The function of tertiary amplifying circuit is magnifying the second-grade signal, for meeting the signal detection and discriminator circuit's requirements. The high gain-band-width amplifier is used and its gain bandwidth product can achieve $20 \mathrm{MHz}$, for this part, noninverting proportional amplifier is employed as shown in Figure 5. The resistances named $R_{6}, R_{7}$ are $1 \mathrm{~K}$ and $100 \mathrm{~K}$ separately and closed loop voltage gain of this circuit is $A=1+R_{7} / R_{6}=101$.

\section{Filter Circuit}

The received signal of SAW usually incorporates some jamming signal, thus these signal should be filtered as far as possible. Nevertheless, it is impossible to remove the jamming signals utterly and the jamming signals can be diminished as far as possible. In this paper, received signal frequency is $1 \mathrm{MHz}$ approximately, and the device composed of operational amplifier, capacitance and others have the characteristics of narrower bandwidth. So the kind of circuit is not suitable for high frequency. However, integrated circuit with inherent high price, thus practical filter consists of capacitance and inductance is preferred.

As shown in Figure 6, the parallel resonant circuit is composed of capacitance and inductance, the resonant frequency is set as $1 \mathrm{MHz}$, and $L_{1}, C_{4}, L_{3}, C_{5}$ comprise the parallel resonant circuit, the T-shaped network and band-pass filtering are all realized.

\section{Automatic Voltage Comparison Circuit}

It is critical to ascertain the arrival time of received signal in the circuit design and it directly affects the measurement accuracy of propagation time of SAW. Therefore, voltage comparing circuit is used to test the received signal in an efficient way and the effective detection threshold is used to satisfy signal's zero crossing point detection. As shown in Figure 7 and Figure 8, the circuit is composed of high-precision zero crossing point comparator LM311 and high speed dual voltage comparator LM393. So the zero signal can be detected by zero crossing point comparator and the high speed dual voltage comparator can achieve the low level and high level signal, and the three output signal are all sent to microcomputer system for logical control.

\section{Conclusion}

In this paper, the received signal is amplified by three levels, and the gains of 


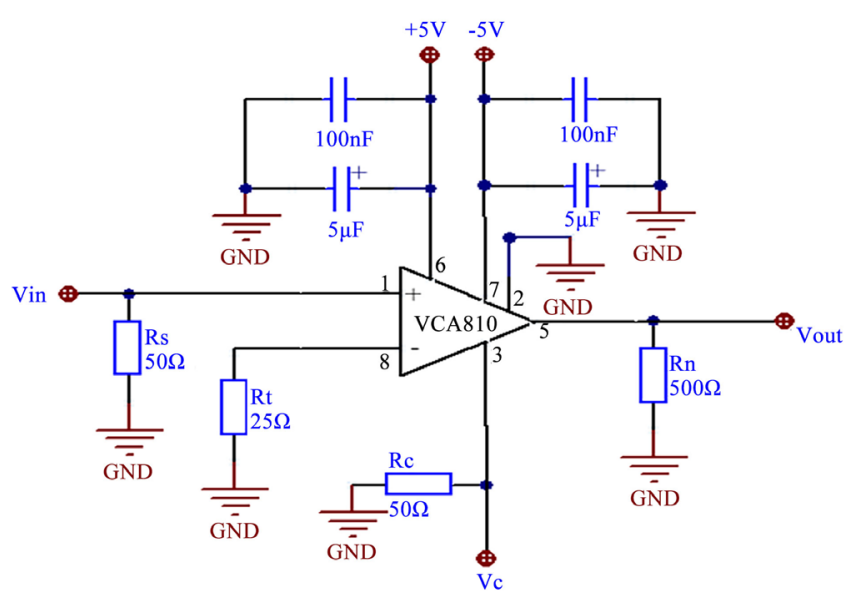

Figure 4. Automatic gain amplifying circuit.

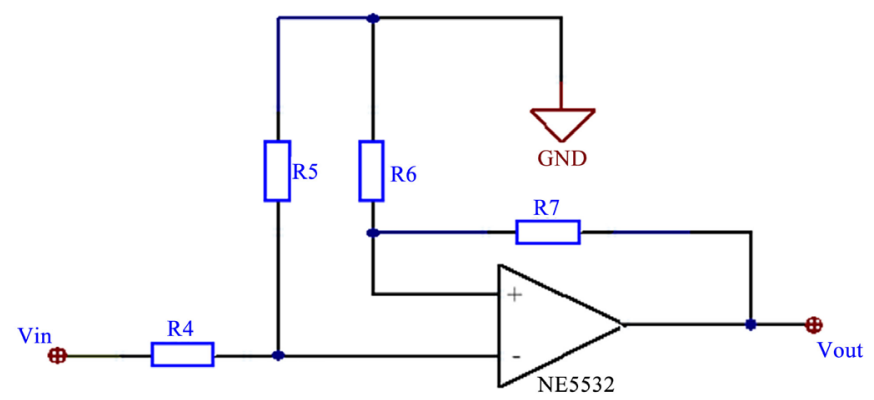

Figure 5. Tertiary amplifying circuit.

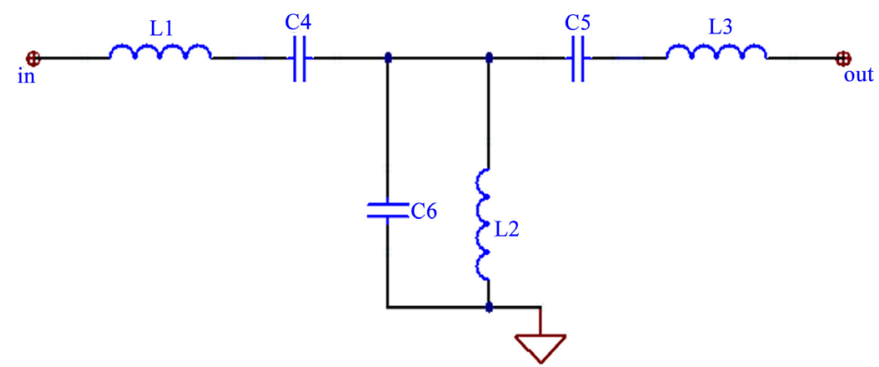

Figure 6. Filter circuit.

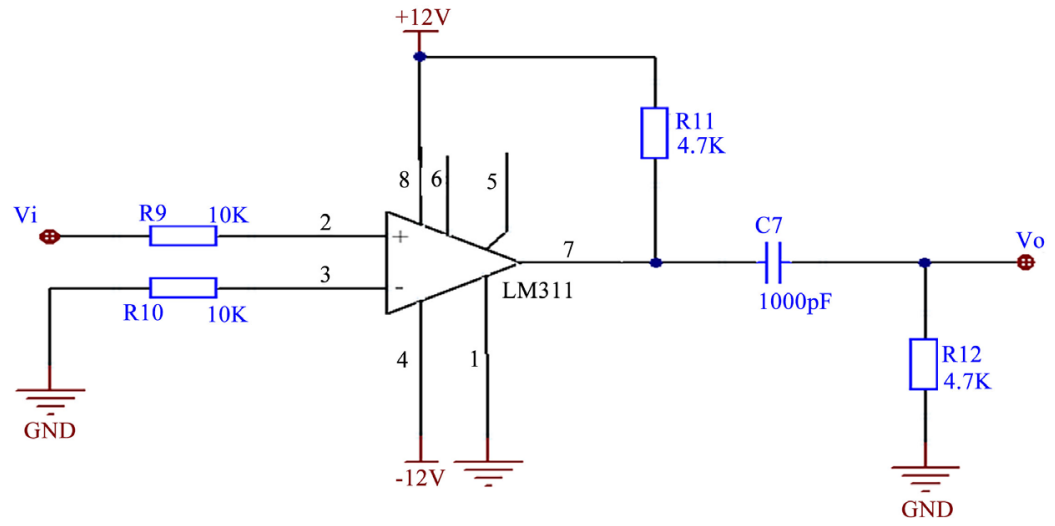

Figure 7. Over-zero comparing circuit. 


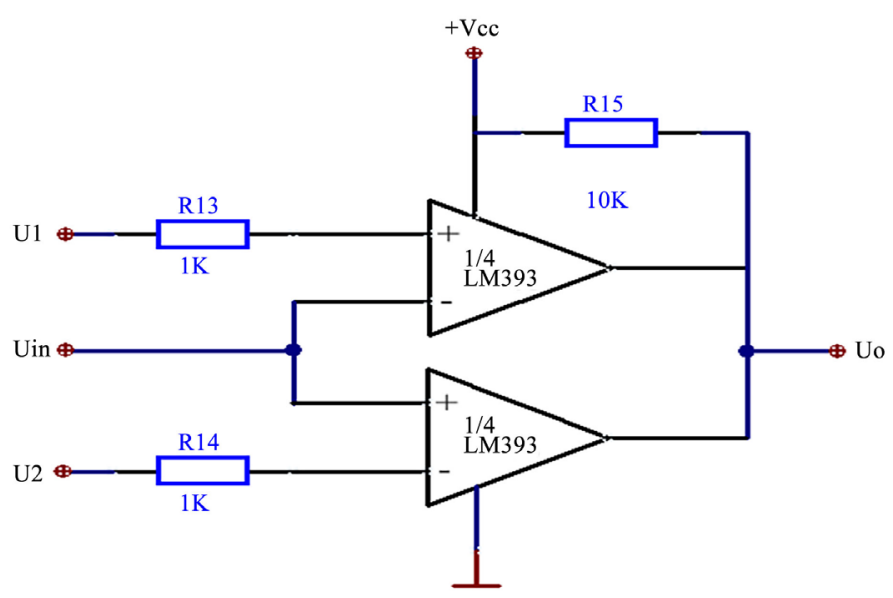

Figure 8. Voltage comparison circuit.

first level and the third level of gains are changeless. The second level of amplification uses voltage controlled chip as automatic gain amplifier, and the received signal peak is amplified, sampled and held, for maintaining steady output voltage. Besides, jamming signals of receiving can be filtered effectively by band-pass filter circuit composed of capacitance and inductance. The electric circuit construction of design is simple and with fewer parts of apparatus, therefore it tends out to be very small and cheap. Moreover, the SAW receiving circuit and trigger circuit can be mixed into sonic sensor, it can be used in oil-water separation system and power window security control in the next step. Undoubtedly, it possesses application prospect in the field of SAW sensors and it also has great values to other fields of SAW application.

\section{Conflicts of Interest}

The authors declare no conflicts of interest regarding the publication of this paper.

\section{References}

[1] Jack, M.A., Grant, P.M. and Collins, J.H. (1980) The Theory, Design, and Applications of Surface Acoustic Wave Fourier-Transform Processors. Proceedings of the IEEE, 68, 450-468. https://doi.org/10.1109/PROC.1980.11674

[2] Campbell, C.K. (1991) Applications of Surface Acoustic and Shallow Bulk Acoustic Wave Devices. Proceedings of the IEEE, 77, 1453-1484. https://doi.org/10.1109/5.40664

[3] Bahl, G., Zehnpfennig, J., Tomes, M., et al. (2011) Stimulated Optomechanical Excitation of Surface Acoustic Waves in a Microdevice. Nature Communications, 2, 403. https://doi.org/10.1038/ncomms1412

[4] Gedge, M. and Hill, M. (2012) Acoustofluidics 17: Theory and Applications of Surface Acoustic Wave Devices for Particle Manipulation. Lab on A Chip, 12, 2998. https://doi.org/10.1039/c2lc40565b

[5] Gao, Z.X., Chen, Z.J., Huang, X., et al. (2014) Design and Implementation of SAW Reader Receiving Circuit. 2013 Symposium on Piezoelectricity, Acoustic Waves, 
and Device Applications, Changsha, 25-27 October 2013, 1-4.

[6] Zhou, M., Zhou, X.D., Wang, R.G., et al. (2007) Study of Weak Signal Receiving Circuit of SAW. Journal of Projectiles Rockets Missiles \& Guidance, 27, 62-64.

[7] Kim, N. and Shin, H. (2011) Active Circuit Approaches for SAW-Less Full-Duplexer Receiver Systems. 2011 IEEE 54th International Midwest Symposium on Circuits and Systems, Seoul, 7-10 August 2011, 1-4. https://doi.org/10.1109/MWSCAS.2011.6026385

[8] Owaki, T. (2010) Surface Acoustic Wave Device, Module Device, Oscillation Circuit, and Method for Manufacturing Surface Acoustic Wave Device. 1-5.

[9] Funami, M., Yokota, Y. and Iioka, K. (2008) Surface Acoustic Wave Resonator, Surface Acoustic Wave Filter and Surface Acoustic Wave Duplexer, and Communications Equipment. 2-4.

[10] Lei, X.T. and Fang, Z.X. (2007) Design of Wireless Microphone's Receiver and Transmitter Circuit Based on the Surface-Acoustic-Wave (SAW) Resonator. Journal of Huaiyin Institute of Technology, 1-3. 